\title{
Circadian Clock in Cell Culture: I. Oscillation of Melatonin Release from Dissociated Chick Pineal Cells in Flow-Through Microcarrier Culture
}

\author{
Linda M. Robertson and Joseph S. Takahashi \\ Department of Neurobiology and Physiology, Northwestern University, Evanston, Illinois 60201
}

\begin{abstract}
The avian pineal gland contains circadian oscillators that regulate the rhythmic release of melatonin. We have developed a dissociated chick pineal cell culture system in order to begin a cellular analysis of this vertebrate circadian oscillator. Dissociated pineal cells maintained in cyclic light conditions (LD 12:12) released melatonin rhythmically. The release of melatonin was elevated during the dark and low during the light. A circadian oscillation of melatonin release persisted for at least 5 cycles in constant darkness with a period close to $24 \mathrm{hr}$; however, there was a gradual damping of the amplitude. Analysis of the rhythm revealed that the observed damping was consistent with either desynchronization of multiple oscillators within the cultures or damping of individual oscillators. The circadian oscillation of melatonin release persisted for up to 4 cycles under conditions of constant light; however, the oscillation was strongly damped and the period of the oscillation was lengthened significantly. Thus, dissociated pineal cells express a persistent circadian oscillation of melatonin release in constant darkness, and properties of this oscillation are modulated by light treatment in vitro. This flow-through cell culture method for dissociated chick pineal cells should provide a useful model for the analysis of a vertebrate circadian system at the cellular level.
\end{abstract}

The behavior and physiology of most organisms are temporally organized in synchrony with the environmental light-dark cycle (Aschoff, 1981; Takahashi and Zatz, 1982). In most cases, daily rhythms in these various processes are generated by an endogenous circadian oscillator or pacemaker (Pittendrigh, 1981). In avian species, behavioral and biochemical evidence suggests that the pineal gland plays a major role in the temporal organization of the animal (Takahashi and Menaker, 1979, 1984a). Pinealectomy abolishes the circadian rhythm of locomotor activity in the house sparrow, Passer domesticus (Gaston and Menaker, 1968). Transplantation of pineal tissue into the anterior eye chamber of a pinealectomized host restores the circadian activity rhythm, and the phase of the restored rhythm is determined by the phase of the donor (Zimmerman and Men-

\footnotetext{
Received Dec. 31, 1987; revised July 16, 1987; accepted July 16, 1987.

This work was supported by NIMH Grant MH-39592, NSF Presidential Young Investigator Award DCB-8451642, and Searle Scholars Award 85-H-107 to J.S.T. We thank Dr. G. D. Niswender for providing the melatonin antibody.

Correspondence should be addressed to Dr. Takahashi, Department of Neurobiology and Physiology, Northwestern University, Hogan Hall, Evanston, IL 60201 .

Copyright (C) 1988 Society for Neuroscience $0270-6474 / 88 / 010012-10 \$ 02.00 / 0$
}

aker, 1979). These results argue that the pineal functions as a circadian pacemaker in sparrows.

In addition to behavioral experiments, an oscillator role for the avian pineal has been tested directly. Because the biosynthetic pathway for the pineal hormone, melatonin, is strongly rhythmic in vivo (Binkley et al., 1973), a number of laboratories have used isolated chick pineal glands in organ culture to determine whether rhythms of serotonin $\mathrm{N}$-acetyltransferase (an enzyme involved in the biosynthesis of melatonin) and melatonin production persist in vitro. While Kasal and colleagues (1979) were able to find 2 cycles of $N$-acetyltransferase activity in constant conditions, Binkley et al. (1978) and Deguchi (1979a) reported that they were unable to find persistent circadian rhythms in constant darkness using static organ culture methods. Long-term perfusion of individual chick pineal glands yielded high resolution in the time domain under cyclic light conditions; however, in constant conditions the rhythm was heavily damped (Takahashi et al., 1980). The difficulty in obtaining persistent oscillations in culture has been a major obstacle in the development of the avian pineal gland as a model system for analyzing the physiological basis of circadian rhythms.

In retrospect, it appears that most of the difficulties in demonstrating persistent circadian oscillations in chick pineal organ cultures can be attributed to inadequate culture conditions. In addition, although organ cultures were sufficient to demonstrate circadian rhythms at a qualitative level, the variability of the rhythms both among and within cultures prevented quantitative analysis. The solution to most of these problems has been achieved by the use of dissociated cell cultures. Deguchi (1979b) first showed that dispersed pineal cell cultures were capable of circadian oscillation for 2 cycles; however, his method still required measurement of $N$-acetyltransferase activity by using a population of cultures to assay the rhythm. We have developed a flow-through cell culture system that allows continuous measurement of a melatonin release rhythm from individual cultures for long periods of time. Furthermore, the variability both within and among cell cultures has been greatly reduced so that quantitative experiments on the period length and phase of the oscillation are now possible. Using this system we have addressed 2 sets of questions in this paper. Do dispersed pineal cell cultures retain the capacity for persistent circadian oscillation? If so, are the characteristics of the oscillation similar to those found in vivo?

\section{Materials and Methods}

Animals

Newly hatched male chicks (Gallus domesticus, white leghorn) were purchased from Combelt hatcheries (Forest, IL). The animals were raised 
under $12 \mathrm{hr}$ light:12 hr dark (LD 12:12) lighting conditions with lights on at 0700 CST for 3-6 weeks prior to study. Food (Purina Chick Startena) and water were available continuously.

\section{Cell culture}

For each primary culture, 10 chicks were sacrificed by decapitation, the pineal glands were removed and collected in sterile PBS. The tissue was transferred to a sterile hood and washed 3 times with Hank's salts solution containing $500 \mu \mathrm{g} / \mathrm{ml}$ gentamicin (US Biochem), $1000 \mathrm{U} / \mathrm{ml}$ penicillin $\mathrm{G}, 1000 \mu \mathrm{g} / \mathrm{ml}$ streptomycin, $25 \mu \mathrm{g} / \mathrm{ml}$ amphotericin B (Irvinc Scientific). The glands were transferred to $4 \mathrm{ml}$ Hank's salts solution containing $1 \mathrm{mg} / \mathrm{ml}$ collagenase (Cooper Biomedical, \#CLS5S01). The tissue was minced and incubated for $30 \mathrm{~min}$ in a $37^{\circ} \mathrm{C}$ shaking water bath. Partially dispersed fragments were triturated with a siliconized, fire-polished Pasteur pipet and allowed to settle for $5 \mathrm{~min}$. The supernatant was transferred to a $15 \mathrm{ml}$ tube and centrifuged for $1 \mathrm{~min}$ at $100 \times g\left(25^{\circ} \mathrm{C}\right)$ to pellet the fragments. The supernatant was transferred to a $15 \mathrm{ml}$ tube and centrifuged for $5 \mathrm{~min}$ at $500 \times g\left(25^{\circ} \mathrm{C}\right)$. The final pellet was resuspended in $4 \mathrm{ml}$ of complete culture medium with the following composition: Medium-199 with Hank's salts and L-glutamine (GIBCO, \#400-1200) supplemented with 10 mM HEPES buffer (US Biochem), 5\% fetal bovine serum (Biologos $\# 1210378$ ), 10\% heat-inactivated horse serum (GIBCO, \#200-6050), $100 \mathrm{U} / \mathrm{ml}$ penicillin G, $100 \mu \mathrm{g} / \mathrm{ml}$ streptomycin, and $0.9 \mathrm{mg} / \mathrm{ml} \mathrm{NaHCO}_{3}$ supplemented for $5 \%$ $\mathrm{CO}_{2}$ atmosphere. The cells were counted with a hemacytometer. A typical dispersal yielded approximately $2 \times 10^{5}$ cells per pineal gland (about $20 \%$ of the cells in a gland). Collagen-coated microcarriers (Pharmacia, Cytodex 3 \#17-0485-01) were hydrated in PBS $(0.02 \mathrm{gm} / \mathrm{ml}$ Cytodex) for at least $3 \mathrm{hr}$ at $25^{\circ} \mathrm{C}$. The microcarriers were sterilized by autoclaving using purified water $(20 \mathrm{~min})$. Following sterilization, the microcarriers were washed 3 times with culture medium. An aliquot $(2.5 \mathrm{ml})$ of a slurry containing a $1: 1$ volume of microcarriers to culture medium was added to the cell suspension. The final volume of the cell suspension was adjusted to $10 \mathrm{ml}$. The cell-bead slurry was divided into two $25 \mathrm{~cm}^{2}$ culture flasks and placed in an incubator at $37^{\circ} \mathrm{C}$ with $95 \%$ air $/ 5 \% \mathrm{CO}_{2}$. The cultures were maintained on LD 12:12 provided by two $7 \mathrm{~W}$ tungsten bulbs and half the medium was changed every $2 \mathrm{~d}$. After 4-5 d in culture, the cells were loaded into the flow-through culture apparatus described below.

\section{Flow-through cell culture technique}

The flow-through cell culture apparatus was composed of 4-20 parallel channels. Each channel included a $13 \mathrm{~mm}$ diameter filter holder (Nuclepore, $\# 420100$ with an $8 \mu \mathrm{m}$ pore size membrane filter), which functioned as a cell chamber and was connected to a syringe pump (Harvard, \#2265) and a fraction collector (ISCO, \#328) using microbore Silastic tubing (Dow Corning, 0.020 in. I.D., 0.037 in. O.D.). The apparatus was sterilized by autoclaving ( $20 \mathrm{~min}$ ). The cell chambers were housed within light-controlled boxes with a $4 \mathrm{~W}$ fluorescent bulb fitted with a Schott KG1 heat filter. The light intensity at the level of the cell chambers was $100-150 \mu \mathrm{W} / \mathrm{cm}^{2}$ measured with a United Detector Technology S350 photometer with a radiometric filter. The entire apparatus was kept in a temperature-controlled chamber at $37^{\circ} \mathrm{C}$ with a humidified atmosphere. Approximately $2 \times 10^{5}$ cells (which is equivalent to about $1 / 5$ pineal on the basis of both the number of cells and the amount of melatonin production) were loaded into each cell chamber using a $3 \mathrm{ml}$ syringe barrel as a funnel. After all the cells had settled into the chamber, the syringe barrel was removed, and the chamber was connected to a $50 \mathrm{ml}$ syringe with Silastic tubing. For flow-through experiments an airequilibrated culture medium was used with the following composition: Medium-199 with Hank's salts and L-glutamine supplemented with 10 mM HEPES buffer, $5 \%$ fetal bovine serum, $10 \%$ heat-inactivated horse serum, and $50 \mu \mathrm{g} / \mathrm{ml}$ gentamicin. The syringes were loaded into the syringe pump, and medium was infused through the cell chambers at a rate of $0.25-0.50 \mathrm{ml} / \mathrm{hr}$ depending on the experiment. A reservoir bottle containing medium to refill the syringes was attached by a 3-way valve and kept in an ice bath until needed. Two-hour fractions were collected from each cell chamber using a fraction collector.

\section{Melatonin radioimmunoassay}

Samples were removed from the fraction collector at 6-15 hr intervals, frozen at $-20^{\circ} \mathrm{C}$, and analyzed at the termination of the experiment. Appropriate volumes $(2-10 \mu \mathrm{l})$ of each sample were assayed for mela- tonin content using radioimmunoassay (Rollag and Niswender, 1976) as described by Takahashi et al. (1980). Iodinated melatonin analog was custom synthesized for use in the radioimmunoassay by Meloy Laboratories (Springfield, VA). Melatonin was purchased from Sigma Normal rabbit gamma glabulin was purchased from Cooper Biomedical Rabbit anti-melatonin antibody (R 1055 pool) was supplied by Dr. G. D. Niswender. The assay has been validated for pineal culture medium without extraction of the samples (Takahashi et al., 1980). No interference from the culture medium was detectable at the sample volumes assayed. The upper and lower limits of the assay were approximately 500 and $1 \mathrm{pg} /$ tubc, respectively. Fifty percent inhibition was typically produced with $18 \mathrm{pg} /$ tube. The coefficients of variation for pooled culture medium quality control samples containing low $(15.0 \mathrm{pg})$, medium $(28.0 \mathrm{pg})$, and high $(84.5 \mathrm{pg})$ levels of melatonin were $8.3,7.3$, and $6.4 \%$ for intraassay and $15.8,18.6$, and $18.3 \%$ for interassay variation, respectively.

\section{Data analysis}

Phase reference points and period length estimates. To determine the period length of the melatonin rhythm, we analyzed 5 phase reference points: peak, trough, half-rise, half-fall, and midpoint of the cycle. All phase points were obtained from the individual records of cell cultures. First, the highest and lowest melatonin values for each cycle were determined. The time points corrcsponding to thcse values represent the peak and trough phase reference points, respectively. The half-rise phase reference point was calculated by interpolating the time point that corresponded to the average melatonin value for the peak and the preceding trough. Similarly, the half-fall phase point was calculated by interpolating the time point that corresponded to the average melatonin value for the peak and the subsequent trough. The midpoint phase reference was determined by averaging the times for the half-rise and half-fall phase reference points. The period length for a given cycle was defined as the difference between 2 of the same consecutive phase reference points. Five different period measurements for an individual cell chamber were made using each of the 5 phase reference points for 4 consecutive cycles in constant darkness beginning the second cycle in culture. The average period length for an individual cell chamber was calculated by averaging the 3 period estimates derived from the 4 cycles. The variance resulting from this mean was the intraindividual variance. The period lengths of cell chambers maintained in the same treatment condition were averaged. The variance resulting from this mean was the interindividual variance. Significance was determined using analysis of variance followed by least-significant difference comparison of treatment means (Sokal and Rohlf, 1981).

Estimation of damping of amplitude of melatonin rhythm. In order to estimate the damping of the circadian melatonin rhythm, we analyzed the amplitude of each cycle in constant darkness. Measurements were made from records of individual cell cultures. The amplitude was defined as the difference between the melatonin value for the peak of each cycle and the melatonin value for the subsequent trough. The amplitude for each cycle was then expressed as a percentage of the amplitude of the first cycle in constant darkness.

\section{Results}

\section{Characteristics of pineal cell cultures on microcarriers}

The cell cultures were visually monitored with phase-contrast microscopy during the $5 \mathrm{~d}$ incubation period prior to use in the flow-through experiments. Several cell types were observed immediately following the dispersal procedure. The majority of the cells were round, phase bright, and approximately 8-10 $\mu \mathrm{m}$ in diameter. The cell surface was relatively smooth and in some cases the nucleus could be seen. On the basis of preliminary cell marker experiments, the majority of the round cells were pinealocytes and the remainder were interstitial cells (J. S. Takahashi and L. M. Robertson, unpublished observations). Occasionally, ependymal cells with beating flagella were found and were presumably derived from the choroid plexus attached to the stalk of the pineal gland. In addition, some red blood cells were present. Immediately after plating, a small percentage of round cells attached to the collagen-coated microcarriers, al- 

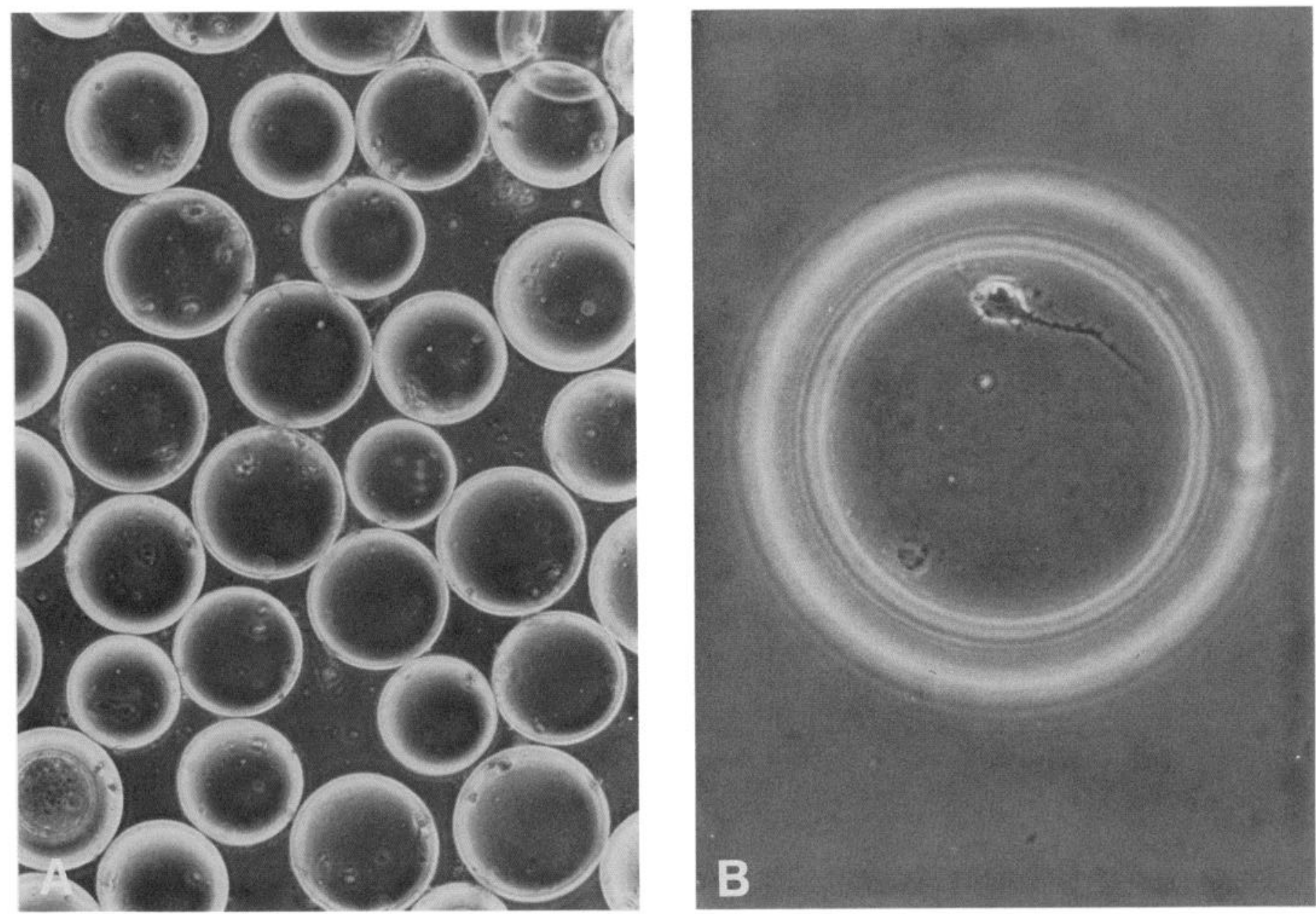

Figure 1. Phase-contrast photomicrographs of dissociated pineal cell cultures on Cytodex microcarriers. A, Cells grown on microcarriers after 7 $\mathrm{d}$ in culture. Original magnification, $100 \times . B$, Single pinealocyte on a microcarrier after $19 \mathrm{~d}$ in culture. Original magnification, $250 \times$.

though the majority of the cells required at least $30 \mathrm{hr}$ for attachment. After $24 \mathrm{hr}$ in culture most of the fibroblastic cells were attached to the plastic bottom of the culture flask. After $48 \mathrm{hr}$ in culture the small round cells flattened somewhat and began to send out processes. While the majority of the round cells were unipolar, some were multipolar. The typical morphological appearance of the dissociated cells on microcarriers is shown in Figure 1. Proliferation of fibroblastic cells was clear after $48 \mathrm{hr}$; however, unlike the round cell types, the majority of the fibroblasts were preferentially attached to the plastic bottom of the culture flask. This differential substrate preference provided a means for enriching the proportion of pinealocytes attached to the microcarriers. When the beads with the round cells attached were loaded into the cell chambers, most of the fibroblasts were left behind.

A preliminary flow-through experiment revealed that the cell cultures required the addition of serum to the medium in order to sustain synthesis and release of melatonin. A population of cells was grown in complete medium containing $10 \%$ horse serum and $5 \%$ fetal bovine serum for $5 \mathrm{~d}$. Cells were then transferred to the flow-through apparatus. Two cell chambers were perfused with medium containing serum, while 3 chambers received no supplement. As illustrated in Figure $2 A$, the release of melatonin in the presence of serum was rhythmic and remained at high levels; however, in the cultures with no supple- ment, the release of melatonin diminished to undetectable values within $24 \mathrm{hr}$.

To investigate the stability of the cell cultures over time, cells were maintained on microcarriers in the incubator for 1 month before initiating a flow-through experiment. Melatonin biosynthesis and release was present even after $30 \mathrm{~d}$ in culture (Fig. $2 B$ ). The time course of release was similar to that observed after only 1 week in culture (Fig. $2 A$ ). This suggests that the expression of rhythmic melatonin release by the cell cultures remains for long periods of time in vitro.

\section{Circadian properties of pineal cell cultures}

To determine whether the dispersed cell cultures were capable of circadian oscillation, we examined melantonin release in constant darkness. In the experiment shown in Figure $3 A$, cultures were exposed to LD 12:12 for $1 \mathrm{~d}$ followed by constant darkness for $4 \mathrm{~d}$. Pineal cells exhibited an increase in melatonin release during the first $12 \mathrm{hr}$ of darkness followed by a decrease during $12 \mathrm{hr}$ of light. This oscillation peristed for at least 4 cycles in constant darkness with a period close to $24 \mathrm{hr}$. However, the amplitude of the rhythm was damped in constant darkness. In contrast, cultures run simultaneously but exposed to LD 12:12 for $5 \mathrm{~d}$ (Fig. $3 B$ ) expressed a high-amplitude oscillation with no damping of the rhythm. The waveform and amplitude of the oscillation were remarkably consistent both among replicate 

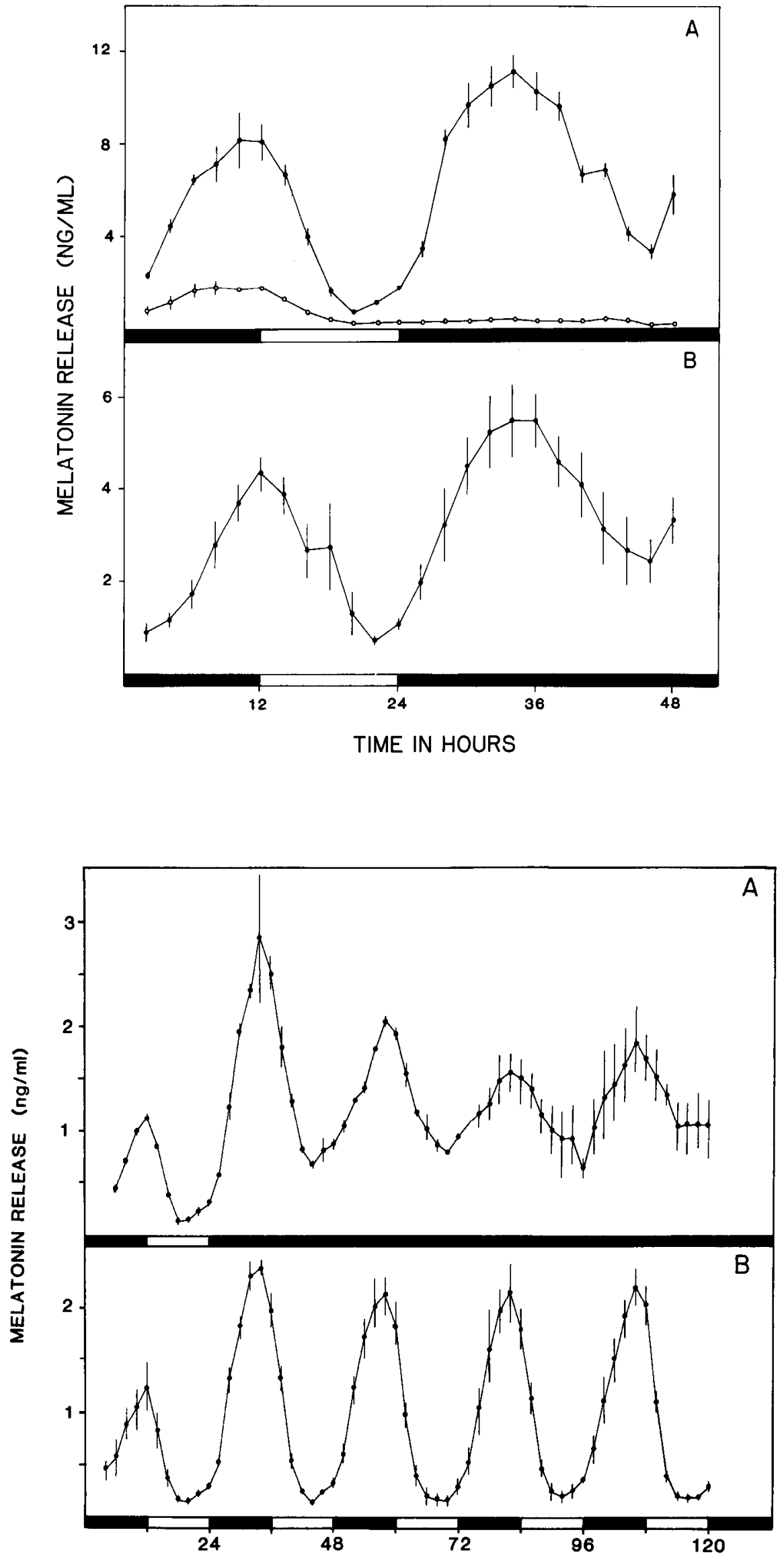

TIME IN HOURS
Figure 2. Effects of culture conditions on melatonin release from dispersed chick pineal cells. The bar at the bottom of each panel indicates the light-dark cyclc. Flow rate in both experiments was $0.5 \mathrm{ml} / \mathrm{hr}$. $A$, Melatonin release from cell cultures maintained in medium with and without serum supplement. Solid circles and bars represent mean and range of melatonin release from duplicate cell chambers containing $10 \%$ horse serum and $5 \%$ fetal calf serum. Open circles and bars represent mean \pm SEM of melatonin release from 3 replicate cell chambers maintained in serum-free medium. $B$, Melatonin release from cell cultures maintained in the incubator under LD 12:12 a month prior to study. Points and bars represent mean \pm SEM of 5 replicate cell chambers.
Figure 3. Rhythmic release of melatonin from dispersed chick pineal cells. The bar at the bottom of each panel indicates the light-dark cycle. Flow rate was $0.5 \mathrm{ml} / \mathrm{hr}$. $A$, Circadian oscillation of melatonin release. Points and bars represent mean and range of duplicate cell chambers exposed to LD 12:12 for one cycle followed by constant darkness for $4 \mathrm{~d}$. B. Diurnal rhythm of melatonin release. Points and hars represent mean \pm SEM of 3 replicate cell chambers exposed to LD 12:12 for the duration of the experiment. 

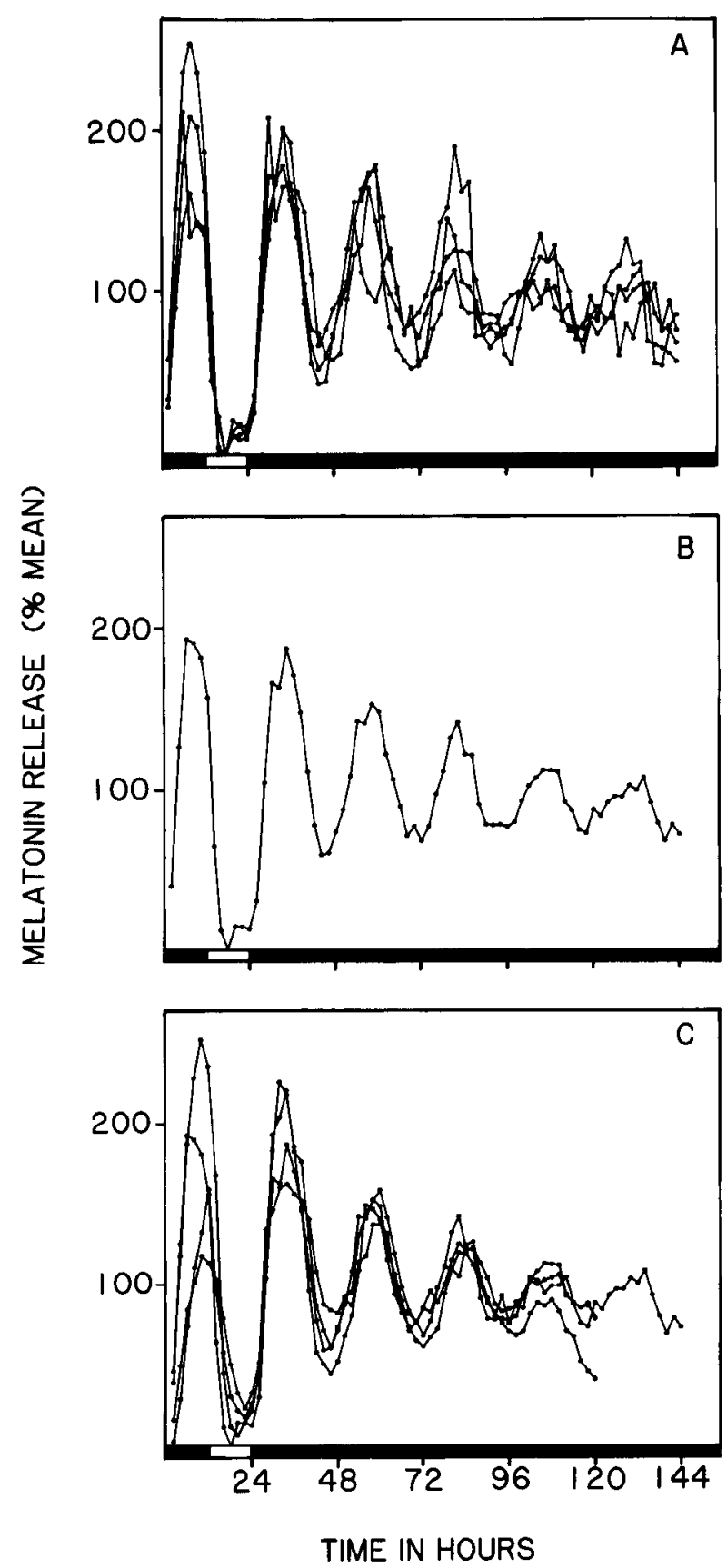

Figure 4. Circadian oscillation of melatonin release from dispersed chick pineal cells. The bar at the bottom of each panel indicates the light-dark cycle. Individual records were normalized in order to compensate for variation in cell number along replicate cell chambers. Normalized values were calculated for each record by dividing the melatonin released per hour during each $2 \mathrm{hr}$ collection interval by the average melatonin released per hour during the entire experimental period (120$144 \mathrm{hr}$ depending on the experiment) and multiplying by $100 \%$. Flow rate was $0.25-0.50 \mathrm{ml} / \mathrm{hr}$ depending on the experiment. $A$, Superimposed individual records from a single experiment demonstrate the variation observed among replicate cell chambers. $B$, Ensemble average of data presented in $A$. $C$, Superimposed ensemble averages from 4 independent experiments demonstrate the variation observed between experiments. Points represent mean of 4-5 replicate cell chambers depending on the experiment. cultures and within consecutive cycles for a single culture. The maintenance of a high-amplitude oscillation in a light-dark cycle indirectly suggests that the dispersed pineal cells are photoreceptive in culture. The oscillation did not appear to be entirely driven by the light-dark cycle, but rather exhibited an anticipatory increase prior to lights out and a decrease prior to lights on. These anticipatory changes in melatonin are consistent with the entrainment of an oscillator. The total amount of melatonin in both conditions did not change. The constant level of melatonin released per cycle suggests that the cells that synthesize melatonin (pinealocytes) probably did not change significantly in cell number or biosynthetic capacity. Visual inspection of sister cultures maintained in the incubator suggests that the number of pineal cells remained relatively constant over time.

To illustrate the consistency of the endogenous pineal melatonin oscillation in these cell cultures, we have compared the melatonin rhythms from individual cell chambers run simultaneously in a single experiment and from different experiments run independently in Figure 4. In this figure we have normalized the amplitude of the records to facilitate comparison among experiments in which variations in cell number per chamber occurred. Figure $4 A$ shows the individual melatonin records from 4 cell chambers run at the same time in a single experiment. These superimposed records illustrate the range of variation seen among replicate cell cultures in a single experiment. Figure $4 B$ shows the ensemble average of the 4 replicates in Figure $4 A$. The melatonin oscillation persists for at least 5 cycles in constant darkness. There is a progressive damping of the amplitude of the rhythm and a progressive increase in the range in variation among replicates with increasing time in constant darkness. In Figure $4 C$ we have compared the ensemble averages from 4 independent experiments (each of which is composed of 4-5 replicates) in order to illustrate the consistency of the melatonin oscillation. Three of the experiments were $120 \mathrm{hr}$ in duration, while the fourth (shown in Fig. $4 B$ ) was $144 \mathrm{hr}$ in duration. Although there are rather large variations in melatonin production during the first $24 \mathrm{hr}$ of the flow-through experiment, the melatonin oscillations are remarkably consistent after the first $24 \mathrm{hr}$. Indeed, the interexperimental variation of the ensemble averages (Fig. $4 C$ ) appears to be less than the interindividual variation of replicates in a single experiment (Fig. $4 A$ ). On the basis of these observations, several features of the melatonin release rhythm in constant darkness emerge. First, the circadian oscillation persists without fail for $\mathbf{4}$ cycles. Second, the amplitude of the rhythm progressively damps and the rate of damping is relatively consistent. Third, the damping progresses towards the mean value of the record with both a decrease in the peak and increase in the trough. Finally, the period length of the rhythm is stable both within and between experiments (quantitative analysis of the period is presented below).

In order to determine how long the endogenous oscillation persisted in culture, we monitored melatonin release for over 1 week in constant darkness. Cell chambers were exposed to LD 12:12 for $24 \mathrm{hr}$ followed by constant darkness for $8 \mathrm{~d}$ (Fig. 5). The melatonin oscillation was detectable for about 5 cycles in constant darkness; however, as observed previously, the rhythm exhibited a gradual decrease in amplitude. As illustrated in Figure 5 , a return to cyclic light conditions reinstated a high-amplitude melatonin rhythm.

As shown in Figure 4, the melatonin oscillation persists in constant darkness; however, the amplitude of the expressed 


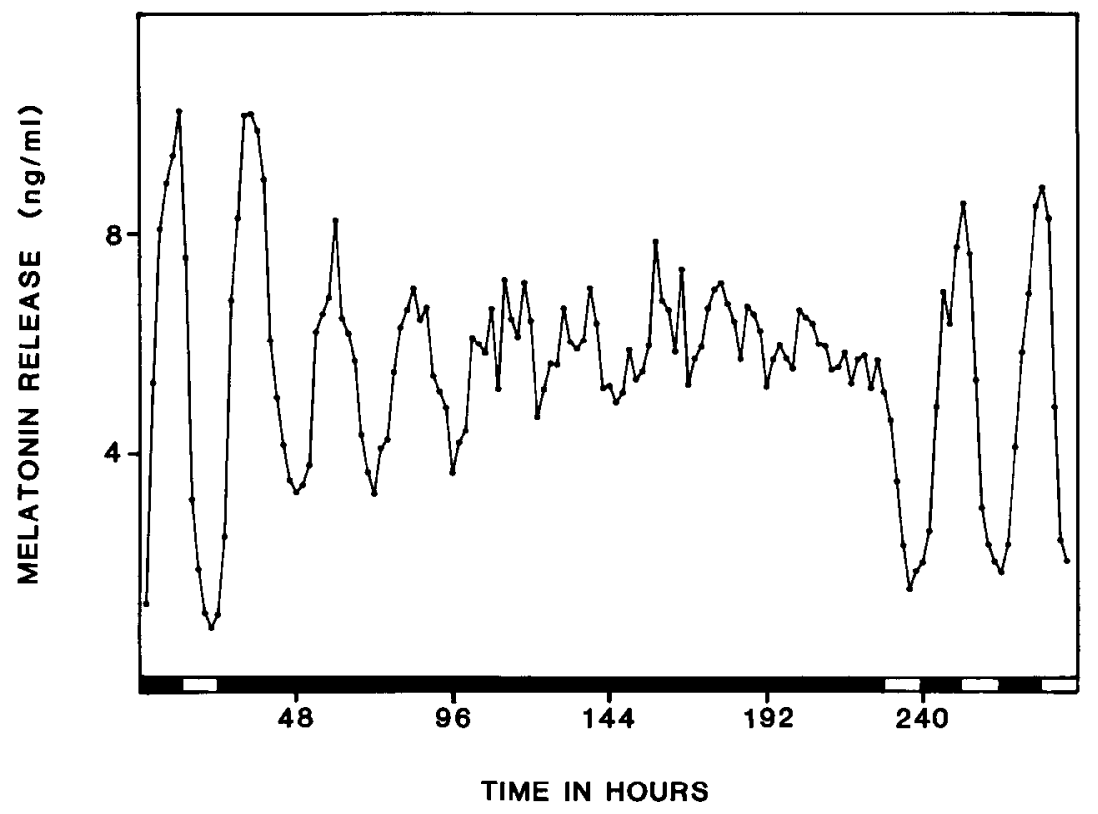

Figure 5. Damping of the melatonin oscillation from dispersed chick pineal cells during long-term constant darkness. The dark bar indicates the lightdark cycle. Points represent melatonin release from one cell chamber. Flow rate was $0.25 \mathrm{ml} / \mathrm{hr}$. Despite a gradual damping of the rhythm during constant darkness, a return to a light-dark cycle (LD 12:12) reinstated a high-amplitude melatonin rhythm. rhythm damps. There are at least 2 possible explanations for this phenomenon: individual pineal oscillators within the cultures may be self-sustained oscillators that gradually desynchronize in constant conditions, leading to overt damping of the rhythm (Takahashi and Menaker, 1984b); alternatively, individual pineal oscillators may be damped oscillators (Enright, 1984). With the first hypothesis, one would assume that the cell cultures are composed of oscillatory cells, each of which has the capability to maintain a self-sustained oscillation of melatonin release. If each oscillator had photic input, the oscillators would be synchronized in cyclic light conditions. However, if there was variability in the endogenous period of each cellular oscillator in constant conditions, this would result in desynchronization of the individual rhythms and apparent damping of the overt melatonin oscillation. The rate of damping would depend mainly on the amount of variability present in the circadian periods of the oscillators in the population. The second hypothesis for the overt damping of the melatonin rhythm has been proposed by Enright (1984). On this hypothesis the overt damping observed in the pineal cell cultures would result from damping of individual oscillators. It is difficult to discriminate between these 2 possibilities. A direct test would involve the measurement of melatonin release in constant conditions from a single pineal cell, which has yet to be accomplished. However, it is possible to examine the implications of these 2 hypotheses.

Analyzing data from Takahashi et al. (1980), Enright (1984) suggested that an extremely broad range of free-running periods, from 12-36 hr, would be required to produce the damping observed in constant conditions assuming a desynchronization model. Because this range of periods deviated significantly from "circadian periodicities," Enright concluded that desynchronization was an unlikely interpretation. The results presented here from cells maintained in constant darkness can be subjected to a similar analysis. The model assumes that the melatonin output from a single cell has a Gaussian distribution in time with a SD on the order of $3 \mathrm{hr}$ and that the distribution of the period lengths in the population of cells is Gaussian. Assuming additivity of variances, each peak would have a variance of $s^{2}=$ $s_{p}{ }^{2}+(n-1)^{2} s_{t}{ }^{2}$, where $s_{p}$ is the SD of the first synchronized melatonin peak in hours, $s_{t}$ is the SD of the individual freerunning periods of the oscillators in the population in hours, and $n$ is the number of cycles in constant darkness. Since the amplitude of a Gaussian distribution is inversely proportional to its $\mathrm{SD}$, it is possible to determine the amplitudes of each subsequent peak in constant darkness. Figure 6 illustrates the damping rates from theoretical curves resulting from this analysis using $\mathrm{SD}\left(s_{t}\right)$ values of $0.5,1,2,3$, and $6 \mathrm{hr}$, respectively. The peak-trough amplitude of each cycle is expressed as a percentage of the amplitude of the first synchronized cycle in constant darkness. Experimental values derived from records in constant darkness are plotted over the theoretical curves in Figure 6 . The experimental data are most closely approximated by the theoretical curve resulting from an SD of $3 \mathrm{hr}$. Assuming no damping occurred, the circadian pcriods of the individual oscillatory cells would then range from 18 to $30 \mathrm{hr}$. A combination of damping and desynchronization would decrease the range of periods required. While there is no empirical evidence available, this range in period lengths seems reasonable for that of a circadian oscillator. Therefore, although it is possible that the overt damping in the melatonin oscillation results from the damping of individual oscillators, theoretical predictions of the desynchronization hypothesis are also consistent with the experimental observations.

\section{Effects of constant light on pineal cell cultures}

We have also investigated the effects of constant illumination on the circadian oscillation of melatonin release. Cell chambers were initially exposed to LD 12:12 followed by $8 \mathrm{~d}$ of bright constant light (intensity at the level of the cell chambers was $100 \mu \mathrm{W} / \mathrm{cm}^{2}$ ). Continuous illumination has been reported to inhibit the nocturnal rise in $\mathrm{N}$-acetyltransferase activity in both organ (Deguchi, 1979a) and cell culture (Deguchi, 1979b). However, as illustrated in Figure 7, constant light did not suppress the nocturnal rise in melatonin release. In spite of a loss of rhythmicity after about 4 cycles, melatonin release was never suppressed to levels similar to those observed during the light 
Figure 6. Desynchronization hypothesis for damping of amplitude in a population of oscillators. Curves represent the theoretical decrease in amplitude of the ensemble rhythm if the SD among the period lengths of component oscillators was $0.5,1,2,3$, or $6 \mathrm{hr}$. All values are expressed as a percentage of the first cycle constant darkness. Points and bars represent amplitude measurements of the melatonin oscillation from dispersed chick pineal cells during successive cycles in constant darkness. Solid circles and bars represent mean \pm SEM of measurements from 15 replicate cell chambers. Open circles and bars represent mean and range of measurements from duplicate cell chambers.

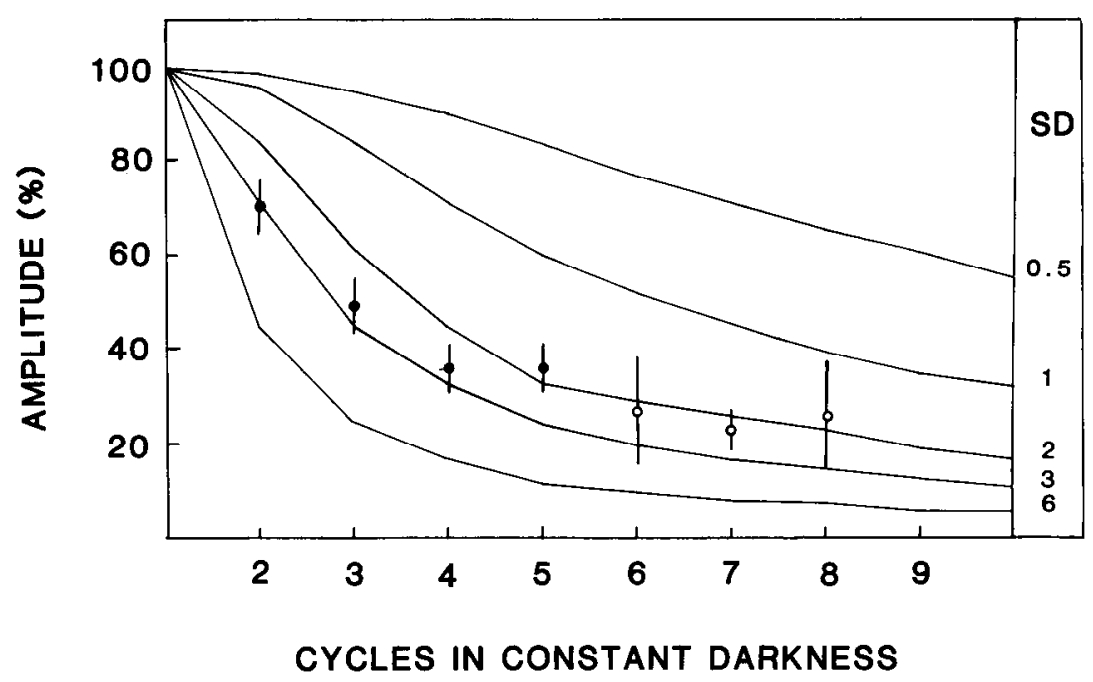

point the steady-state period of the circadian oscillator was 24.4 hr in constant darkness (Table 1). Constant light treatment significantly lengthened the period to $26.4 \mathrm{hr}$. The lengthening of period by constant light treatment is opposite that expected on the basis of empirical data for diurnal birds as described by Aschoff's rule, which shows that constant light treatment shortens the freerunning period in passerine birds (Daan and Pittendrigh, 1976). In intact chickens, however, brain temperature (Aschoff and von Saint Paul, 1973), activity, and oviposition rhythms (Cain and Wilson, 1972) did not show a significant change in period with increasing light intensities over the limited range tested. Therefore, domestic chickens might represent an exception to Aschoff's rule.

\section{Discussion}

As a first step in beginning a cellular analysis of the circadian pacemaking system in the avian pineal, we have addressed 2 sets of questions. Do dispersed pineal cell cultures retain the capacity for circadian oscillation? If so, are the characteristics of the oscillation similar to those found in organ culture and in vivo? In the flow-through cell culture system, dispersed pineal cells clearly retain the capacity for circadian oscillation. In spite of a gradual damping of the amplitude, the oscillation is detectable for at least 5 cycles in constant darkness. Furthermore, the consistency of the oscillation both among replicates within a single experiment and among independent experiments is remarkably good. The consistency of the melatonin oscillation greatly exceeds that observed in previous in vitro experiments using chick pineal, making it possible to perform quantitative experiments in which the period and phase of the oscillation serve as end points.

The expression of a circadian oscillation in melatonin release from dispersed pineal cells implies that the capacity for circadian rhythmicity does not require normal tissue organization. Our results support previous observations of rhythms in $\mathrm{N}$-acetyltransferase activity from dispersed pineal cell cultures (Deguchi, 1979b). Both Deguchi's results and the results reported here suggest that the circadian oscillation in the pineal may be a cellular property, although a definitive demonstration awaits further experimentation. If the capacity for circadian oscillation is indeed a cellular property, it is of interest to determine the trough, $3.81 \pm 0.39 \mathrm{hr}(X \pm \mathrm{SEM})$. The midpoint phase reference point yielded the smallest variance. Using this reference 


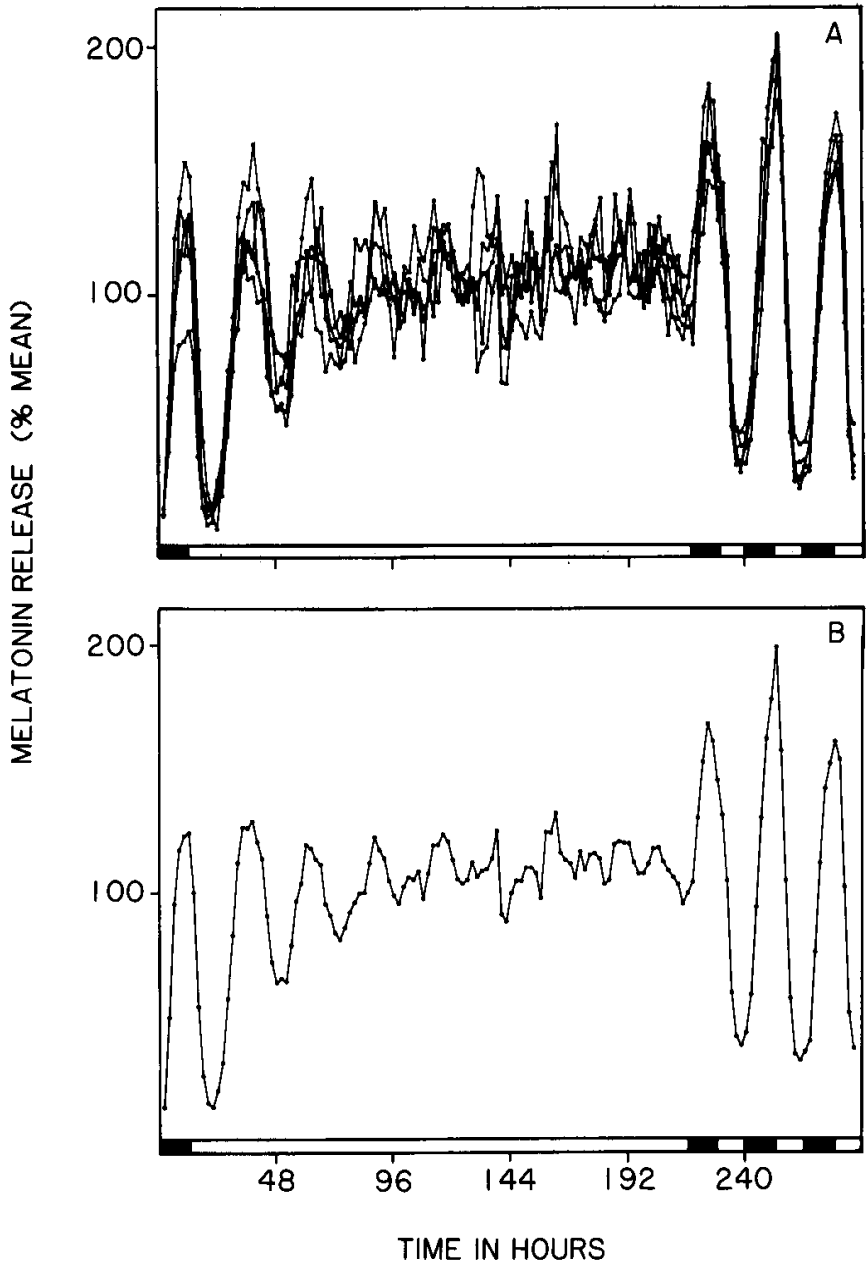

Figure 7. Effects of constant illumination on the circadian oscillation of melatonin release from dispersed chick pineal cells. The bar at the bottom of each panel indicates the light-dark cycle. Individual records were normalized in order to compensate for variation in cell numbers among replicate cell chambers as described in Figure 5. Flow rate was $0.25 \mathrm{ml} / \mathrm{hr}$. $A$, Superimposed individual records from 5 replicate cell chambers exposed to $1 \mathrm{~d}$ of LD 12:12 followed by $8 \mathrm{~d}$ of constant light and $3 \mathrm{~d}$ of $L D$ 12:12. $B$, Ensemble average of individual records presented in $A$. Note the persistence of the oscillation for several cycles in constant light despite a general damping of the rhythm.
Table 1. Average period length and variation of melatonin rhythms from chick pineal cells in different light conditions

\begin{tabular}{llllr} 
Treatment & $\begin{array}{l}\text { Period } \\
\text { lcngth } \\
(\mathrm{hr})^{a}\end{array}$ & $\begin{array}{l}\text { Interin- } \\
\text { dividual } \\
\text { SD }(\mathrm{hr})\end{array}$ & $\begin{array}{l}\text { Intraindi- } \\
\text { vidual SD } \\
(\text { mean } \pm \text { SEM) }\end{array}$ & $N^{c}$ \\
\hline LD 12:12 & 23.9 & 0.25 & $0.47 \pm 0.09$ & 3 \\
Constant darkness & 24.4 & 1.15 & $1.51 \pm 0.18$ & 21 \\
Constant light & $26.4^{d}$ & 1.17 & $1.98 \pm 0.48$ & 5
\end{tabular}

${ }^{a}$ For each culture, the period length was calculated from the average of the periods from the first 4 cycles ( 3 period values) in the light condition using the midpoint as a phase reference.

${ }^{b}$ Intraindividual SD was calculated from the period estimates from the 4 cycles used to estimate period length.

' $N$ refers to the number of pineal cell cultures in each condition.

${ }^{d}$ Analysis of variance of period length revealed a significant difference among treatment means $(p<0.003, d f=26)$. The constant light treatment group differed from both the constant darkness and LD 12:12 groups (least significant difference test, $p<0.025$ ).

cell types present in the cultures. There are few reports describing avian pineal cell cultures. However, the structure of the intact pineal gland is relatively well described (Boya and $\mathrm{Za}$ morano, 1975; Omura, 1977; Oshima and Matsuo, 1984; Sato and Wake, 1984). A connective tissue sheath surrounds the pineal in chickens. The gland itself is composed of many lobules separated by septa of collagen penetrating inward from the capsule. Ultrastructural studies of cell types indicate that the organ consists mainly of photoreceptorlike cells, secretory cells, and interstitial or ependymal cells (Bischoff, 1969). The outer-segment-like processes of the photoreceptorlike cells resemble degenerated retinal cones (Collin and Oksche, 1981). In cell culture, the morphological appearance of the cells is different from that found in the intact tissue. As a result, distinctions of the various cells types in culture based on morphological descriptions of the intact gland are difficult. We believe, however, that the use of appropriate immunocytochemical cell markers will enable us to identify the various cell types in the cultures in future work.

Although the ultrastructural evidence for photoreceptors in the chick pineal has been equivocal, there is clear functional evidence for photoreceptive properties of the avian pineal. $\mathrm{Di}$ rect illumination of the cultured gland suppresses the nocturnal

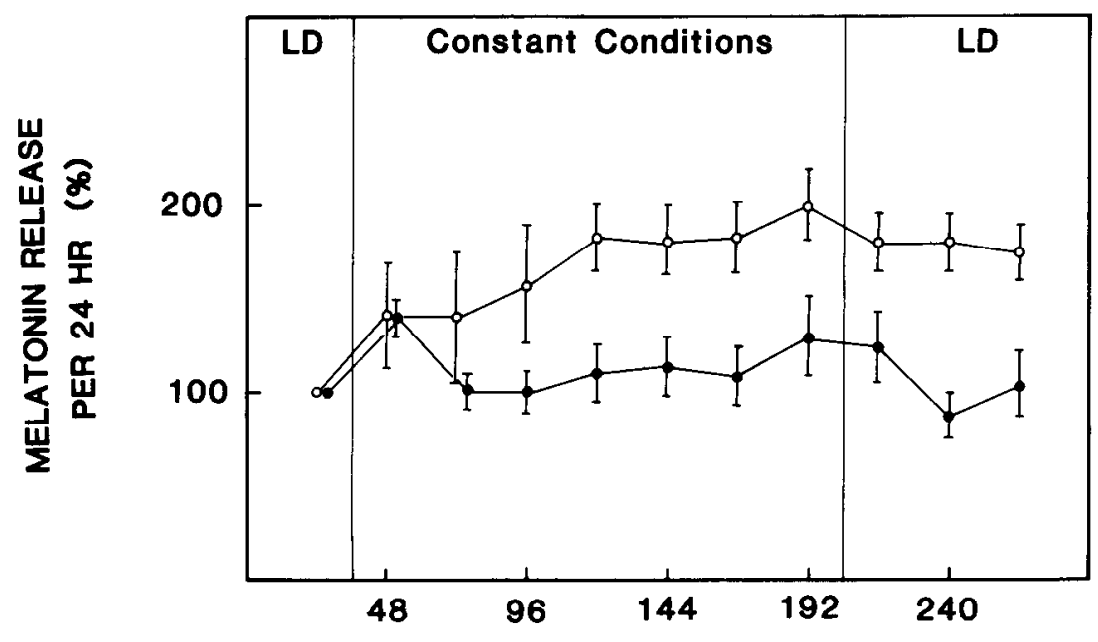

TIME IN HOURS
Figure 8. Daily melatonin release in constant conditions expressed as a percentage of the first cycle on LD 12:12. Solid circles and bars indicate mean \pm SEM of 4 replicate cell chambers maintained in constant darkness. Open circles and bars indicate mean \pm SEM of 5 replicate cell chambers maintained in constant light. The daily melatonin release in constant darkness was relatively constant while the daily melatonin release in constant light increased nearly 2 -fold. 
rise in $\mathrm{N}$-acetyltransferase activity and acute exposure to light during the night rapidly inhibits $N$-acetyltransferase activity and melatonin release (Deguchi, 1979a; Wainwright and Wainwright, 1980; Hamm et al., 1983). The action spectrum for the suppression of the nocturnal rise in $N$-acetyltransferase activity resembles the absorbance spectrum of rhodopsin (Deguchi, 1981). Furthermore, rhodopsin immunoreactivity has been detected in cells surrounding the lumen of the gland (Vigh et al., 1982; Takahashi, H. Hamm, and E. Mezey, in preparation). Thus, like the retina, the avian pineal gland appears to be a specia!ized structure for the reception, transduction, and transmission of photic information.

In many circadian systems, exposure to constant light damps the amplitude of the overt rhythm. This effect of constant light could reflect either acute inhibition of the output of the system or a direct effect on the circadian oscillator that controls the output. In the chick pineal the effects of constant light have been studied both in vivo and in vitro. Ralph et al. (1975) maintained male chicks in constant light and determined $N$-acetyltransferase activity after 2 weeks. Long-term exposure to constant light in vivo abolished the $N$-acetyltransferase activity rhythm, although the level of enzyme activity remained rather high. The effects of constant light during the initial days of light treatment have also been examined in vivo. Male chicks were maintained in LD 14:10 for several weeks then transferred to constant light. Surprisingly, a rhythm in $\mathrm{N}$-acetyltransferase activity persisted during the first $3 \mathrm{~d}$ in constant light in 2 independent studies (Wainwright and Wainwright, 1981; Tanabe et al., 1983). In addition, the peak time of enzyme activity was delayed by 4-5 $\mathrm{hr}$ on the first day of constant light exposure relative to that seen on a light-dark cycle. The effects of constant light on the chick pineal gland have also been investigated in culture. Exposure of cultured chick pineal glands to constant light resulted in suppression of $N$-acetyltransferase activity (Binkley et al., 1978; Deguchi, 1979a; Wainwright and Wainwright, 1980). However, in most cases, a rhythm in enzyme activity was still detectable in constant light. Limitations of the culture systems prohibited the measurement of the rhythm for more than 1 cycle. Since an initial delay of the rhythm was obscrved during constant light in vivo, it is possible that a component of the lightinduced suppression of $N$-acetyltransferase activity observed in organ culture is due to a delay in the phase of the oscillation and not entirely the result of acute suppression of the enzyme activity. We were able to monitor melatonin release from dispersed chick pineal cells for more than 1 week in constant light. Constant light did not suppress melatonin production. The oscillation persisted, although heavily damped, for about 3 or 4 cycles. As found in vivo, the phase of the first peak during constant light was delayed nearly $5 \mathrm{hr}$ relative to dark controls. Thus, the effects of constant illumination on dispersed pineal cell cultures are very similar to those reported in vivo. In both cases an oscillation of melatonin or $N$-acetyltransferase activity persists for at least 3 cycles after transfer to constant light. However, after long-term exposure to constant light the rhythms in both dispersed cell cultures and intact animals are abolished. Because constant light lengthened the period of the melatonin rhythm in cell cultures, this treatment must act upon the circadian oscillator at some level. Whether the abolition of the rhythm after long-term treatment is due to an effect related to the period-lengthening effects of light on the oscillator or to a direct effect on the output of the oscillator cannot be distin- guished. The apparent lack of acute suppression of melatonin release during constant light both in the intact animal and in dispersed cell cultures is a surprising result. The absence of an acute inhibitory effect of light on pineal melatonin after previous light exposure (i.e., the $12 \mathrm{hr}$ of light during the day) may indicate either that the photopigment mediating acute inhibition is bleached or that the pineal photoreceptors lose sensitivity by a process similar to adaptation.

In summary, dispersed chick pineal cell cultures contain circadian oscillators that express a persistent rhythm of melatonin release in constant darkness. In contrast to previous in vitro systems for the avian pineal, the melatonin oscillation is extremely consistent both within an individual experiment and among independent experiments, so that quantitative experiments on the period and phase of the oscillation are now possible. In the following paper (Robertson and Takahashi, 1988), we use this system to analyze the photic entrainment and phaseshifting behavior of this vertebrate circadian oscillator in vitro.

\section{References}

Aschoff, J. (1981) Handbook of Behavioral Neurobiology, Vol. 4, Biological Rhythms, Plenum, New York.

Aschoff, J., and U. von Saint Paul (1973) Circadian rhythms of brain temperature in the chicken, measured at different levels of constant illumination. Jpn. J. Physiol. 23: 69-80.

Binkley, S., S. E. MacBride, D. C. Klein, and C. L. Ralph (1973) Pineal enzymes: Regulation of avian melatonin synthesis. Science 181:273285.

Binkley, S., J. B. Riebman, and K. B. Reilly (1978) The pineal gland: A biological clock in vitro. Science 202: 1198-1201.

Bischoff, M. B. (1969) Photoreceptoral and secretory structures in the avian pineal organ. Ultrastruct. Res. 28: 16-26.

Boya, J., and L. Zamorano (1975) Ultrastructural study of the pineal glands of chicken (Gallus gallus). Acta Anat. 92: 202-226.

Cain, J. R., and W. O. Wilson (1972) A test of the circadian rule of Aschoff with chicken hens. J. Interdiscipl. Cycle Res. 3: 77-85.

Collin, J. P., and A. Oksche (1981) Structural and functional relationships in the nonmammalian pineal gland. In The Pineal Gland, Vol. I. Anatomy and Biochemistry, R. J. Reiter, ed., pp. 27-67, CRC Press, Boca Raton, FL.

Daan, S., and C. S. Pittendrigh (1976) A functional analysis of circadian pacemakers in nocturnal rodents. III. Heavy water and constant light: Homeostasis of frequency? J. Comp. Physiol. 106: 267290.

Deguchi, T. (1979a) Circadian rhythm of serotonin N-acetyltransferase activity in organ culture of chicken pineal gland. Science 203: $1245-1247$

Deguchi, T. (1979b) A circadian oscillator in cultured cells of chicken pineal gland. Nature 282: 94-96.

Deguchi, T. (1981) Rhodopsin-like photosensitivity of isolated chicken pineal gland. Nature 290: 706-707.

Enright, J. T. (1984) Mutual excitation of damped oscillators and selfsustainment of circadian rhythms. In Mathematical Models of the Circadian Sleep-Wake Cycle, M. C. Moore-Ede and C. A. Czeisler, eds., pp. 1-15, Raven, New York.

Gaston, S., and M. Menaker (1968) Pineal function: The biological clock in the sparrow? Science 160: 1125-1127.

Hamm, H., J. S. Takahashi, and M. Menaker (1983) Light-induced decrease of serotonin $\mathrm{N}$-acetyltransferase activity and mclatonin in the chicken pineal gland and retina. Brain Res. 266: 287-293.

Kasal, C. A., M. Menaker, and J. R. Perez-Polo (1979) Circadian clock in culture: N-Acetyltransferase activity in chick pineal glands oscillates in vitro. Science 203: 656-658.

Omura, Y. (1977) Ultrastructural study of embryonic and post-hatching development in the pineal organ of the chicken (Brown Leghorn, Gallus domesticus). Cell Tissue Res. 183: 255-271.

Oshima, K., and S. Matsuo (1984) Functional morphology of the pineal gland in young chickens. Anat. Anz. (Jena) 156: 407-418.

Pittendrigh, C. S. (1981) Circadian systems: Entrainment. In Hand- 
book of Behavioral Neurobiology, Biological Rhythms, Vol. 4, J. Aschoff, ed., pp. 57-80, Plenum, New York.

Ralph, C. L., S. Binkley, S. E. MacBride, and D. C. Klein (1975) Regulation of pineal rhythms in chickens: Effect of blinding, constant dark, and superior cervical ganglionectomy. Endocrinology 97: 13731378.

Robertson, L. M., and J. S. Takahashi (1988) Circadian clock in cell culture: II. In vitro photic entrainment of melatonin oscillation from dissociated chick pineal cells. J. Neurosci. 8: 22-30.

Rollag, M. D., and G. D. Niswender (1976) Radioimmunoassay of serum concentrations of melatonin in sheep exposed to different lighting regimes. Endocrinology 98: 482-489.

Sato, T., and K. Wake (1984) Regressive post-hatching development of acetylcholinesterase-positive neurons in the pineal organs of $\mathrm{Co}$ turnix coturnix japonica and Gallus gallus. Cell Tissue Res. 237:269275.

Sokal, R. R., and F. J. Rohlf (1981) Biometry, pp. 239-244, Freeman, San Francisco.

Takahashi, J. S., and M. Menaker (1979) Physiology of avian circadian pacemakers. Fed. Proc. 38: 2583-2588.

Takahashi, J. S., and M. Menaker (1984a) Circadian rhythmicity: Regulation in the time domain. In Biological Regulation and Development, Vol. 3B, K. R. Yamamoto and R. F. Goldberger, eds., pp. 285-303, Plenum, New York.

Takahashi, J. S., and M. Menaker (1984b) Multiple redundant cir- cadian oscillators within the isolated avian pineal gland. J. Comp. Physiol. 154: 435-440.

Takahashi, J. S., and M. Zatz (1982) Regulation of circadian rhythmicity. Science 217: 1104-1111.

Takahashi, J. S., H. Hamm, and M. Menaker (1980) Circadian rhythms of melatonin release from individual superfused chicken pineal glands in vitro. Proc. Natl. Acad. Sci. USA 77: 2319-2322.

Tanabe Y., D. Osamu, and T. Nakamura (1983) Ontogenesis and photoperiodic regulation of pineal hormone synthesis in the chicken, Gallus domesticus. In Avian Endocrinology: Environmental and Ecological Perspectives, S. Mikami et al., eds., pp. 217-227, Japan Sci. Soc. Press, Tokyo.

Vigh, B., I. Vigh-Teichmann, P. Rohlich, and B. Aros (1982) Immunoreactive opsin in the pineal organ of reptiles and birds. A. Mikrosk.-anat. Forsch. (Leipzig) 96: 113-129.

Wainwright, S. D., and L. K. Wainwright (1980) Regulation of cycle in chick pineal serotonin $\mathrm{N}$-acetyltransferase activity in vitro by light. J. Neurochem. 35: 451-457.

Wainwright, S. D., and L. K. Wainwright (1981) Regulation of chick pineal serotonin- $\mathrm{N}$-acetyltransferase activity by light and extra-pineal factor(s). In Pineal Function, C. D. Matthews and R. F. Seamark eds., pp. 199-209, Elsevier/North-Holland Biomedical, Amsterdam Zimmermann, N. H., and M. Menaker (1979) The pineal: A pacemaker within the circadian system of the house sparrow. Proc. Natl. Acad. Sci. USA 76: 999-1003. 